\title{
Club-guessing and non-structure of trees
}

\author{
by \\ Tapani Hyttinen (Helsinki)
}

\begin{abstract}
We study the possibilities of constructing, in ZFC without any additional assumptions, strongly equivalent non-isomorphic trees of regular power. For example, we show that there are non-isomorphic trees of power $\omega_{2}$ and of height $\omega \cdot \omega$ such that for all $\alpha<\omega_{1} \cdot \omega \cdot \omega, E$ has a winning strategy in the Ehrenfeucht-Fraïssé game of length $\alpha$. The main tool is the notion of a club-guessing sequence.
\end{abstract}

In this paper we study the problem of constructing strongly equivalent non-isomorphic models. In the 60's, the problem was studied in connection with the arising interest in infinitary languages. The idea was to study the relation between isomorphism and elementary equivalence in infinitary logics. Well known contributions were made e.g. by C. Karp and M. Morley. These studies were continued in the 70's, e.g. by M. Nadel and J. Stavi. See [NS] for more on the early history. In the 80 's, the infinitary languages were revisited in Helsinki and the question was raised again (see e.g. [Hu]). By this time, S. Shelah had come to the field with his non-structure theorems, in which the conclusion was that the theory has rather equivalent but non-isomorphic models. Because of this, the question was revised. We want to know under what conditions a given class of structures has very equivalent non-isomorphic models and how equivalent the models can be; see e.g. $[\mathrm{HT}]$ (complete first-order theories), [MO] (p-groups) or [EFS] ( $\aleph_{1}$-separable groups). Here the idea is to show that one cannot find simple invariants for the models in the class. Thus, when we are able to show that in the class there are very equivalent non-isomorphic models, the result is called a strong non-structure theorem. In these results the equivalence is usually measured by the length of the Ehrenfeucht-Fraïssé games in which $E$ has a winning

2000 Mathematics Subject Classification: Primary 03C75; Secondary 03E05.

Key words and phrases: trees, non-structure, guessing clubs.

Partially supported by the Academy of Finland, grant 40734. The author wishes to express his gratitude to J. Väänänen and J. Kennedy for their valuable comments and to the referee for his suggestions. 
strategy (see e.g. [HS]). We write $\mathcal{A} \equiv_{t}^{\lambda} \mathcal{B}$ if $E$ has a winning strategy in the Ehrenfeucht-Fraïssé game of length $t$ in which the player $A$ chooses sequences of length $<\lambda$. In the strongest non-structure theorems we had to assume that $\kappa^{<\kappa}=\kappa$ to get a required pair of models in cardinality $\kappa$ and we were able to show that at least some assumptions are needed in such theorems (see [HST]). Weaker, but still strong, non-structure theorems were obtained under weaker cardinal arithmetical assumptions (see e.g. [HS]). However, it was soon noticed that without the assumption $\kappa^{\omega}=\kappa$, strong non-structure theorems were hard to get. Explicitly this was pointed out in $[\mathrm{Tu}]$, where the following two propositions were proved:

(i) there are non-isomorphic models $\mathcal{A}$ and $\mathcal{B}$ of power $\omega_{1}$ such that $\mathcal{A} \equiv_{\alpha}^{2} \mathcal{B}$ for all $\alpha<\omega \cdot \omega$

(ii) if the majorant property holds, then there are non-isomorphic models $\mathcal{A}$ and $\mathcal{B}$ of power $\omega_{1}$ such that $\mathcal{A} \equiv_{\alpha}^{2} \mathcal{B}$ for all $\alpha<\omega \cdot \omega \cdot \omega$.

Here the majorant property is the same as $*_{\omega}^{\omega_{1}}$ (see Section 1 below). Then ten years passed without any improvements concerning the problem.

In this paper we try to improve the two propositions above. First, we want the models to be structures familiar from other connections; in fact, we are interested in trees of restricted height. This is an important class of models. From the point of view of Shelah's non-structure theory, the class of trees of low height is probably the most important one among the classes of structures of a specific type. Trees of low height are used as skeletons in Ehrenfeucht-Mostowski model constructions in the case of unsuperstable theories. In $[\mathrm{Tu}]$ the models were specially designed for the result. Secondly, we make changes to the assumptions (e.g. replace $\omega_{1}$ by $\omega_{2}$ ) and try to get stronger conclusions. For trees of low height (compared to their size), in many cases we solve the question of how equivalent non-isomorphic models the class contains (measured by $\equiv^{2}$ and proved in ZFC).

The following two results on trees were proved before (in ZFC): M. Morley showed that there are $\omega_{2}, \omega_{1}$-trees $\mathcal{A}$ and $\mathcal{B}$ of power $\omega_{1}$ such that $\mathcal{A} \neq \mathcal{B}$ and $\mathcal{A} \equiv{ }_{\omega}^{\omega_{1}} \mathcal{B}$ (i.e. $\mathcal{A} \equiv \mathcal{B}\left(L_{\infty \omega_{1}}\right)$; see $[\mathrm{NS}]$ and notice that $\mathcal{A} \not \equiv_{\omega+1}^{2} \mathcal{B}$ ). S. Shelah showed that for all regular $\kappa>\omega$, there are $\kappa^{+}, \omega+1$-trees $\mathcal{A}$ and $\mathcal{B}$ of power $\kappa$ such that $\mathcal{A} \neq \mathcal{B}$ and $\mathcal{A} \equiv \equiv_{\omega}^{\kappa} \mathcal{B}$ (see [Sh1] and notice that $\left.\mathcal{A} \not \equiv_{\omega+1}^{\kappa} \mathcal{B}\right)$. Notice also that without cardinal arithmetical assumptions, Shelah's construction does not generalize to higher trees $\left(\kappa^{<\omega}=\kappa\right)$. On the other hand, Shelah showed the result not only for trees but for all unsuperstable theories $T(\kappa>|T|)$.

Our main tool will be the use of club-guessing. Shelah's original reason to study club-guessing was to prove non-structure theorems. Since then there has been other applications for club-guessing in model theory (see e.g. [KS]). The most famous ones are in set theory, of course. We will introduce a 
generalization of the majorant property mentioned above. It can be seen as a variant of club-guessing principles.

In the first section we study the provability of the generalization of the majorant property. In the second section we apply the principle to get strong non-structure theorems. The constructions in this section use ideas from the model constructions of $[\mathrm{Tu}]$. Most of our results are proved without any assumptions on the cardinal arithmetic, and in the rest the conclusions follow from assumptions much weaker than $\kappa^{\omega}=\kappa$.

1. Club-guessing. We define three combinatorial principles. The first two are modifications of S. Shelah's club-guessing ([Sh5]) and the last is a generalization of $\mathrm{H}$. Tuuri's majorant property $([\mathrm{Tu}])$.

1.1. Definition. Assume $\kappa$ is a regular cardinal and $\alpha<\kappa$.

(i) By $\checkmark_{\alpha}^{\kappa}$ we denote the following principle: There are $C_{i} \subseteq \kappa, i<\kappa$, such that

(a) $C_{i}$ is a closed subset of $\bigcup\left\{\beta+1 \mid \beta \in C_{i}\right\}$ and $\operatorname{otp}\left(C_{i}\right)=\alpha$,

(b) for all cub $C \subseteq \kappa$ there is $i<\kappa$ such that $C_{i} \subseteq C$.

(ii) By $\mathbf{\square}_{\alpha}^{\kappa}$ we denote the following principle: There are $C_{i} \subseteq \kappa, i<\kappa$, such that

(a) $C_{i}$ is a closed subset of $\bigcup\left\{\beta+1 \mid \beta \in C_{i}\right\}$ and $\operatorname{otp}\left(C_{i}\right)=\alpha$,

(b) for all cub $C \subseteq \kappa$ there is $i<\kappa$ such that $C_{i} \subseteq C$,

(c) for all $\beta<\kappa$, the cardinality of the set $\left\{C_{i} \cap \beta \mid i<\kappa, C_{i} \cap \beta\right.$ $\left.\neq C_{i}\right\}$ is $<\kappa$.

(iii) By $*_{\alpha}^{\kappa}$ we denote the following principle: There are $f_{i}: \alpha \rightarrow \kappa, i<\kappa$, such that for all $g: \kappa^{<\alpha} \rightarrow \kappa$ there is $i<\kappa$ for which $g\left(\left(f_{i}(\gamma)\right)_{\gamma<\beta}\right)<f_{i}(\beta)$ for all $\beta<\alpha$.

Notice that $\kappa^{\lambda}=\kappa$ implies $*_{\alpha}^{\kappa}$ and $\boldsymbol{\vee}_{\alpha}^{\kappa}$, for all $\alpha<\lambda^{+}$, and that all the properties are preserved under ccc-forcing. So con(ZFC) implies e.g. $\operatorname{con}\left(\mathrm{ZFC}+\mathrm{MA}+\neg \mathrm{CH}+\forall \alpha<\omega_{1}\left(*_{\alpha}^{\omega_{1}}\right)\right)$.

1.2. TheOREM ([Sh3]). Assume that $\kappa$ and $\xi$ are regular cardinals and $\xi^{+}<\kappa$. Then $\nabla_{\xi}^{\kappa}$ holds.

The following theorem is implicitly proved in a very early (9/91) manuscript of [Sh5]. Since [Sh5] has not appeared yet and a bit additional work is needed, we will repeat the proof:

1.3. Theorem. For all regular $\kappa>\omega_{1}$ and $\alpha<\kappa, \mathbf{\square}_{\alpha}^{\kappa^{+}}$holds.

Proof. By [Sh2], Lemma 4.4, there are $S_{\xi}, \omega<\operatorname{cf}(\xi) \leq \xi<\kappa$, and $C_{\delta}^{\xi}$, $\delta \in S_{\xi}$, such that:

(1) Each $S_{\xi}$ is a subset of $\left\{\delta<\kappa^{+} \mid \operatorname{cf}(\delta)<\kappa\right\}$. 
(2) $C_{\delta}^{\xi} \subseteq\{\gamma<\delta \mid \operatorname{cf}(\gamma)<\kappa\}$ is a closed subset of $\delta$ of power $<\kappa$ and unbounded if $\delta$ is a limit ordinal.

(3) For all $\xi<\kappa$ and $\delta \in S_{\xi}$, if $\gamma \in C_{\delta}^{\xi}$, then $\gamma \in S_{\xi}$ and $C_{\gamma}^{\xi}=C_{\delta}^{\xi} \cap \gamma$.

(4) For all $\delta<\kappa^{+}$with $\operatorname{cf}(\delta)<\kappa$, there is a cub $E \subseteq \kappa$ such that for all $\xi \in E$, if $\operatorname{cf}(\xi)>\omega$, then $\delta \in S_{\xi}$.

(5) If $\delta<\kappa^{+}$is limit, $C \subseteq\{\gamma<\delta \mid \operatorname{cf}(\gamma)<\kappa\}$ is of power $<\kappa$ and $\xi$ is large enough (and $\operatorname{cf}(\xi)>\omega$ ), and $\delta \in S_{\xi}$, then $C \subseteq C_{\delta}^{\xi}$.

Notice that (4) is claim (a) in the proof of Lemma 4.4 in [Sh2], and (5) follows by induction on $\delta$ from the definition of $C_{\delta}^{\xi}$ as follows (we assume that the reader is familiar with the proof of Lemma 4.4 of [Sh2]): The case $\operatorname{cf}(b(\delta)) \neq \operatorname{cf}(\xi)$ is immediate by (ii) and (iii) of the definition of $D_{\alpha}^{i}$, and in the case $\operatorname{cf}(b(\delta))=\operatorname{cf}(\xi)$ notice that we can restrict the intersection to those $W$ which are subsets of some (fixed) $W^{*}$ such that there is $\xi^{*}<\kappa$ for which the following holds: If $\delta^{\prime} \in W^{*}$ and $\xi>\xi^{*}$ then $\delta^{\prime} \in S_{\xi}$ implies $C \cap \delta^{\prime} \subseteq C_{\delta^{\prime}}^{\xi}$.

Claim 1. For all $\delta^{*}<\kappa$ there is $\xi<\kappa$ for which the following holds: for all cub $C \subseteq \kappa^{+}$, there is $\delta \in S_{\xi}$ such that $\operatorname{otp}\left(C_{\delta}^{\xi} \cap C\right)>\delta^{*}$.

Proof. Assume the claim is not true for $\delta^{*}$. For all $\xi<\kappa$, choose $C_{\xi}$ which witnesses the failure of the claim for $\xi$. Let $C=\bigcap_{\xi<\kappa} C_{\xi}, C^{*}=$ $\{\gamma \in C \mid \operatorname{cf}(\gamma)<\kappa\}$ and choose $\delta \in C^{*}$ so that $\operatorname{otp}\left(C^{*} \cap \delta\right)$ is limit, $<\kappa$ and $>\delta^{*}$. By (4) and (5) above, we can find $\xi<\kappa$ so that $\delta \in S_{\xi}$ and $C^{*} \cap \delta \subseteq C_{\delta}^{\xi}$, a contradiction. $\mathbf{m l a i m} 1$

Let $\xi<\kappa$ be as in Claim 1 for $\delta^{*}=\alpha$. We write $S=S_{\xi}$ and $C_{\delta}=C_{\delta}^{\xi}$ $(\delta \in S)$.

Claim 2. There is a cub $C$ such that for all cub $E$ there is $\delta \in S$ for which $C_{\delta} \cap C \subseteq E$ and $\operatorname{otp}\left(C_{\delta} \cap C\right)>\alpha$.

Proof. Assume not. For all $i<\kappa$ choose cub $E_{i}$ so that

(a) $E_{i} \subseteq C^{i}=\bigcap_{j<i} E_{j}$,

(b) for all $\delta \in S, C_{\delta} \cap C^{i} \nsubseteq E_{i}$ or $\operatorname{otp}\left(C_{\delta} \cap C^{i}\right) \leq \alpha$.

Let $C=\bigcap_{i<\kappa} E_{i}$ and choose $\delta \in S$ so that $\operatorname{otp}\left(C_{\delta} \cap C\right)>\alpha$. On the other hand, $\left(C_{\delta} \cap E_{i}\right)_{i<\kappa}$ is a decreasing sequence and so there is $i<\kappa$ such that $C_{\delta} \cap E_{j}=C_{\delta} \cap E_{i}$ for all $i<j<\kappa$, in particular $C_{\delta} \cap E_{i+1}=C_{\delta} \cap C^{i+1}$, which means $C_{\delta} \cap C^{i+1} \subseteq E_{i+1}$, a contradiction. 'Claim 2

Let $S^{*}=\left\{\delta \in S \mid \operatorname{otp}\left(C_{\delta} \cap C\right)>\alpha\right\}$, where $C$ is as in Claim 2. For all $\delta \in S^{*}$, we define $C_{\delta}^{*}$ to be the set of the first $\alpha$ elements of $C_{\delta} \cap C$. Now these witness that $\mathbf{\square}_{\alpha}^{\kappa^{+}}$holds: (a) and (b) of Definition 1.1(ii) are clear. Also (c) holds since for all $\beta<\kappa^{+}$and $\delta \in S^{*}$, if $C_{\delta} \cap \beta \neq C_{\delta}$, then there is $\gamma \leq \beta$ such that up to one element, $C_{\delta}^{*} \cap \beta=C_{\gamma}^{\xi} \cap C$. 
1.4. Lemma. Assume that $\kappa$ is a regular cardinal, $\alpha<\kappa$ and $\mu^{|\beta|}<\kappa$ for all cardinals $\mu<\kappa$ and $\beta<\alpha$. Then $\boldsymbol{\alpha}_{\alpha}^{\kappa}$ implies $\mathbf{\square}_{\alpha}^{\kappa}$. In particular, $\boldsymbol{\vee}_{\omega}^{\kappa}$ implies $\mathbf{\square}_{\omega}^{\kappa}$ for all regular $\kappa>\omega$.

Proof. Immediate.

1.5. Lemma. $\square_{\omega_{1}}$ implies $\boldsymbol{\square}_{\alpha}^{\omega_{2}}$ for all $\alpha<\omega_{1}$.

Proof. Just repeat the proof of Theorem 1.3: Fix $\alpha$ and let $C_{\delta}, \delta<\omega_{2}$, witness $\square_{\omega_{1}}$.

Claim. There is a cub $C \subseteq \omega_{2}$ for which the following holds: for all cub $E \subseteq \omega_{2}$ there is $\delta<\omega_{2}$ such that $C_{\delta} \cap C \subseteq E$ and $\operatorname{otp}\left(C_{\delta} \cap C\right)>\alpha$.

Proof. Assume not. Choose $E_{i}, i<\omega_{1}$, so that $E_{i} \subseteq C^{i}=\bigcap_{j<i} E_{j}$ and for all $\delta<\omega_{2}, C_{\delta} \cap C^{i} \nsubseteq E_{i}$ or otp $\left(C_{\delta} \cap C^{i}\right) \leq \alpha$. Let $C=\bigcap_{i<\omega_{1}} E_{i}$ and $\delta \in C$ be such that $\operatorname{otp}(C \cap \delta)=\omega_{1}$. Then $\operatorname{otp}\left(C \cap C_{\delta}\right)=\omega_{1}$ and there is $\gamma \in C \cap C_{\delta}$ such that $\beta=\operatorname{otp}\left(C \cap C_{\delta} \cap \gamma\right)$ is a limit and $>\alpha$. Since $C_{\delta} \cap \gamma=C_{\gamma}$, we have $\beta=\operatorname{otp}\left(C \cap C_{\gamma}\right)$. Since $\left|C_{\gamma}\right|<\omega_{1}$, there is $i<\omega_{1}$ such that $C^{i} \cap C_{\gamma}=C \cap C_{\gamma}$, a contradiction. - ${ }_{\text {Claim }}$

Let $C$ be as in the Claim and define $C_{\delta}^{*}$ to be the set of the first $\alpha$ elements of $C_{\delta} \cap C$ (if $\operatorname{otp}\left(C_{\delta} \cap C\right) \leq \alpha$, then $C_{\delta}^{*}$ is undefined). Clearly these satisfy (a) and (b) from Definition 1.1(ii). Also (c) is satisfied since for all $\delta<\omega_{2}$ and $\beta<\omega_{2}$, if $C_{\delta}^{*} \cap \beta \neq C_{\delta}^{*}$, then there is $\gamma \leq \beta$ such that up to a finite number of elements, $C_{\delta}^{*} \cap \beta=C_{\gamma} \cap C$.

1.6. Lemma. Assume $\kappa$ is a regular uncountable cardinal and $\alpha<\kappa$ is a limit ordinal. Then $\mathbf{\square}_{\alpha}^{\kappa}$ implies $*_{\alpha}^{\kappa}$. (For successor $\alpha, \mathbf{\square}_{\alpha+1}^{\kappa}$ implies $*_{\alpha}^{\kappa}$.)

Proof. Let $C_{i}, i<\kappa$, witness $\mathbf{\square}_{\alpha}^{\kappa}$. For all $i<\kappa$, let $f_{i}^{\prime}: \alpha \rightarrow \kappa$ be the increasing enumeration of $C_{i}$ and let $f_{i}: \alpha \rightarrow \kappa$ be such that $f_{i}(\gamma)=f_{i}^{\prime}(\gamma+1)$ for all $\gamma<\alpha$. We show that these witness $*_{\alpha}^{\kappa}$. For this let $g: \kappa^{<\alpha} \rightarrow \kappa$ be arbitrary. We let $C \subseteq \kappa$ be the set of all $\gamma$ such that the following holds: if for some $i<\kappa, \eta=f_{i} \mid \delta, \delta<\alpha$ and $\sup (\operatorname{rng}(\eta))<\gamma$, then $g(\eta)<\gamma$. By (c) in the definition of $\boldsymbol{\square}_{\alpha}^{\kappa}, C$ is cub and so there is some $i<\kappa$ such that $C_{i} \subseteq C$. But then $g\left(\left(f_{i}(\gamma)\right)_{\gamma<\beta}\right)<f_{i}(\beta)$ for all $\beta<\alpha$.

1.7. Lemma. (i) If $\boldsymbol{\gamma}_{\alpha}^{\kappa}$ holds and $\beta<\alpha \cdot \omega$, then ${ }_{\beta}^{\kappa}$ holds.

(ii) If $*_{\alpha}^{\kappa}$ holds and $\beta<\alpha \cdot \omega$, then $*_{\beta}^{\kappa}$ holds.

Proof. We prove (i), the other claim is similar. Let $D_{i}, i<\kappa$, exemplify that ${ }_{\alpha}^{\kappa}$ holds. We let $C_{i}, i<\kappa$, enumerate all $C$ such that

(i) $\operatorname{otp}(C)=\beta$,

(ii) $C$ is the closure of an initial segment of $\bigcup_{k<n} D_{i_{k}}$, where $i_{0}, \ldots, i_{n-1}$ $<\kappa$ are such that $\sup \left(D_{k}\right)<\min \left(D_{m}\right)$ for all $k<m<n$.

Clearly these show that $\boldsymbol{}_{\beta}^{\kappa}$ holds. 
The following conclusion lists all the club-guessing facts that we shall need.

1.8. Conclusion. (i) $*_{n}^{\omega_{1}}$ is true for all $n<\omega$.

(ii) $*_{\alpha}^{\omega_{2}}$ is true for all $\alpha<\omega \cdot \omega$.

(iii) $*_{\alpha}^{\kappa^{+}}$is true for all regular $\kappa>\omega_{1}$ and all $\alpha<\kappa$.

(iv) If $\square_{\omega_{1}}$ holds, then $*_{\alpha}^{\omega_{2}}$ holds for all $\alpha<\omega_{1}$.

Proof. (i) is trivial and the remaining are immediate by Theorems 1.2 and 1.3 and Lemmas $1.4-1.7$.

The poset in the proof of the following theorem is due to J. Baumgartner $[\mathrm{Ba}]$.

1.9. Theorem. $*_{\omega}^{\omega_{1}}$ is independent of $Z F C$.

Proof. Since $*_{\omega}^{\omega_{1}}$ is implied by $\mathrm{CH}$, it is enough to prove the consistency of $\neg * \omega_{\omega}^{\omega_{1}}$. We say that $f: \omega_{1} \rightarrow \omega_{1}$ is cub if it is strictly increasing and continuous. We let $Q$ be the set of all finite $p$ such that $p \subseteq f$ for some cub $f$. We order $Q$ by inclusion.

Claim 1. $Q$ is proper.

Proof. Let $p \in Q$ and $M \prec H_{\kappa}$ be such that $p, Q \in M$. Let $\alpha=\omega_{1} \cap M$ and $q=p \cup\{(\alpha, \alpha)\}$. Clearly,

$$
\gamma+\alpha=\alpha \quad \text { for all } \gamma<\alpha,
$$

and so it is easy to see that $q \in Q$. Let $D \in M$ be dense and $q^{\prime} \leq q$. Then $q^{\prime}\left\lceil\alpha \in M\right.$ and so there is $p^{\prime} \in M \cap D$ such that $p^{\prime} \leq q^{\prime}\left\lceil\alpha\right.$. By $(*), p^{\prime} \cup q^{\prime} \in Q$ and clearly $p^{\prime} \cup q^{\prime} \leq q^{\prime}, p^{\prime}$.

Let $G$ be $Q$-generic over $V$ and $F=\bigcup G$. Let $g: \omega_{1}^{<\omega} \rightarrow \omega_{1}$ be one-to-one and such that for all $\eta, g(\eta)>\max \{\eta(n) \mid n \in \operatorname{dom}(\eta)\}$ and if $\eta \subseteq \xi$ then $g(\eta) \leq g(\xi)$. Define $G=F \circ g$.

Claim 2. If $f: \omega \rightarrow \omega_{1}$ is such that $f \in V$, then there is $n<\omega$ for which $G\left((f(i))_{i<n}\right)>f(n)$.

Proof. For a contradiction, assume $p$ forces that $G\left((f(i))_{i<n}\right) \leq f(n)$ for all $n<\omega$. Let $\beta_{n}=f(n)$ and $\alpha_{n}=g\left((f(i))_{i<n}\right)$. We may assume that $\sup _{n<\omega} \beta_{n}=\sup _{n<\omega} \alpha_{n}$, because otherwise $\sup _{n<\omega} \beta_{n}<\sup _{n<\omega} \alpha_{n}$, which is impossible since $F(\alpha) \geq \alpha$ for all $\alpha<\omega_{1}$. Let $\alpha=\sup _{n<\omega} \alpha_{n}$ and $h: \omega_{1} \rightarrow \omega_{1}$ be a cub such that $p \subseteq h$. Clearly we may assume that $h(\alpha)=\alpha(h(\alpha)>\alpha$ gives an easy contradiction $)$. Let $\beta=\max (\operatorname{dom}(p) \cap \alpha)$. For a contradiction, it is enough to find a continuous strictly increasing $h^{\prime}:(\alpha-\beta) \rightarrow\{h(\gamma) \mid \beta \leq \gamma<\alpha\}$ such that $\bigcup\left\{h^{\prime}(\gamma) \mid \beta \leq \gamma<\alpha\right\}=\alpha$ and $h^{\prime}\left(\alpha_{n}\right)>\beta_{n}$ for some $n<\omega$. So it is enough to prove the following subclaim: 
SubClaim. Assume $\alpha<\omega_{1}$ is a limit ordinal and $\left(\alpha_{n}\right)_{n<\omega}$ and $\left(\beta_{n}\right)_{n<\omega}$ are strictly increasing cofinal sequences in $\alpha$. Then there is a continuous strictly increasing function $h: \alpha \rightarrow \alpha$ such that $h\left(\alpha_{n}\right)>\beta_{n}$ for some $n<\omega$.

Proof (by induction on $\alpha$ ). If $\gamma+\alpha=\alpha$ for all $\gamma<\alpha$, then the claim is clear. So we may assume that $\alpha=\beta+\gamma$ for some $\beta, \gamma<\alpha$. But now the claim follows from the induction assumption. - Subclaim \& Claim 2

We will use the theory of $\omega_{2}$-pic forcing. For the definition of $\omega_{2}$-pic see [Sh4], Chapter VIII. However, we will not need the definition, just general results from [Sh4].

Claim 3. $Q$ is $\omega_{2}$-pic.

Proof. By Claim 1 and [Sh4], VIII, Lemma 2.5, it is enough to show that $|Q|<\omega_{2}$, which is trivial. Claim 3

For all $i<\omega_{2}$, let $P_{i}$ be the forcing notion obtained by iterating $Q i$ times using countable support. By $G_{i}$ we denote an (arbitrary) $P_{i}$-generic set over $V$. By Claims 1 and 3, for all $i<\omega_{2}, Q_{i}$ is proper and $\omega_{2}$-pic in $V\left[G_{i}\right]$ (assuming no cardinals are collapsed) and so by [Sh4], III, Theorem 3.2 , and VIII, Lemmas 2.4 and 2.3, assuming $\mathrm{CH}, P_{\omega_{2}}$ is proper and has $\omega_{2}$-cc. In particular, $P_{\omega_{2}}$ does not collapse any cardinals. We show that $*_{\omega}^{\omega_{1}}$ fails in $V\left[G_{\omega_{2}}\right]$.

Assume not. Let $f_{i}, i<\omega_{1}$, witness $*_{\omega}^{\omega_{1}}$. Since $P_{\omega_{2}}$ has $\omega_{2}$-cc, there is $\alpha<\omega_{2}$ such that $f_{i} \in V\left[G_{\omega_{2}}\lceil\alpha]\right.$ for all $i<\omega_{1}$. By Claim 2, there is $g$ : $\omega_{1}^{<\omega} \rightarrow \omega_{1}$ in $V\left[G_{\omega_{2}}\lceil(\alpha+1)]\right.$ such that for all $i<\omega_{1}, g\left(\left(f_{i}(k)\right)_{k<n}\right)>f_{i}(n)$ for some $n<\omega$, a contradiction.

1.10. Question. Does $*_{\alpha}^{\omega_{2}}$ hold for $\omega \cdot \omega \leq \alpha<\omega_{1}$ ?

Notice that by Conclusion 1.8(iv), at least a Mahlo is needed to make $*_{\alpha}^{\omega_{2}}$ fail for some $\alpha<\omega_{1}$.

2. Non-structure of trees. In this section we apply the club-guessing principles from the previous section to get strongly equivalent non-isomorphic trees without any assumptions on the $\beth$-function. We start with a result that sets some limits on what is possible to prove. Let $\kappa$ be a cardinal and $\alpha$ an ordinal. By a $\kappa^{+}, \alpha$-tree we mean a downward closed subtree of $\kappa^{<\alpha}$ (or a tree isomorphic to one).

Warning: This definition differs from the usual definition of $\lambda, \beta$-tree, but it is convenient for our purposes.

2.1. Theorem ([HT], $[\mathrm{HS}])$. Assume $\kappa=\mu^{+}, \mu$ regular, $\kappa \in I[\kappa]$ and $\alpha<\kappa$. If $\mathcal{A}$ and $\mathcal{B}$ are $\kappa^{+}, \alpha$-trees and $\mathcal{A} \equiv{ }_{\mu \cdot \alpha}^{\kappa} \mathcal{B}$, then $\mathcal{A} \cong \mathcal{B}$. (In fact, if $\mu=\omega$, then $\mathcal{A} \equiv_{\mu \cdot \alpha}^{2} \mathcal{B}$ suffices and if $\mu=\lambda^{+}$, then $\mathcal{A} \equiv_{\mu \cdot \lambda \cdot \alpha}^{2} \mathcal{B}$ suffices.)

Proof. This is essentially proved in [HS]. 
If $\mathcal{A}$ is a $\kappa^{+}, \alpha$-tree and $\eta \in \mathcal{A}$, then by $\mathcal{A}\lceil\eta$ we mean the subtree of $\mathcal{A}$ of all $\xi \in \mathcal{A}$ such that $\xi \geq \eta$.

2.2. Definition. Let $\mathcal{A}$ and $\mathcal{B}$ be $\kappa^{+}, \alpha$-trees and $\kappa=\mu^{+}$. We say that $(\mathcal{A}, \mathcal{B})$ is a $\left(\kappa^{+}, \alpha\right)$-homogeneous pair of trees if the following holds:

(i) if $\gamma<\alpha$ and $\eta \in \kappa^{<\gamma}$, then $\eta \in \mathcal{A}$ iff $\eta \in \mathcal{B}$,

(ii) if $\eta \frown(\delta) \in \mathcal{A}$ and length $(\eta)+2<\alpha$, then $\mid\{\gamma|\mathcal{B}|(\eta \frown(\gamma)) \cong$ $\mathcal{A} \uparrow(\eta \frown(\delta))\} \mid \geq \mu$

(iii) if $\eta \frown(\delta) \in \mathcal{B}$ and length $(\eta)+2<\alpha$, then $\mid\{\gamma \mid \mathcal{A} \uparrow(\eta \frown(\gamma)) \cong$ $\mathcal{B}\lceil(\eta \frown(\delta))\} \mid \geq \mu$.

Item (ii) in the following lemma puts some limits on what can be proved by the model constructions of $[\mathrm{Tu}]$.

2.3. Lemma. Assume $\kappa=\mu^{+}$and $\alpha<\kappa$.

(i) If $*_{\alpha}^{\kappa}$ holds, then there is a $\left(\kappa^{+}, \alpha+1\right)$-homogeneous pair $(\mathcal{A}, \mathcal{B})$ of trees such that $|\mathcal{A}|=|\mathcal{B}|=\kappa, \mathcal{A} \neq \mathcal{B}$ and $\mathcal{A} \equiv_{\alpha}^{\kappa} \mathcal{B}$. (In fact, $\mathcal{A} \equiv_{\mu \cdot \alpha}^{2} \mathcal{B}$.)

(ii) If $*_{\alpha}^{\kappa}$ fails, $\alpha$ is a limit ordinal and $(\mathcal{A}, \mathcal{B})$ is a $\left(\kappa^{+}, \alpha+1\right)$-homogeneous pair of trees, then $\mathcal{A} \cong \mathcal{B}$.

Proof. (i) Let $f_{i}, i<\kappa$, exemplify that $*_{\alpha}^{\kappa}$ holds. For all $\eta \in \kappa^{\leq \alpha}$, we define $P(\eta)$ as follows:

(a) If length $(\eta)=0$, then $P(\eta)=0$.

(b) Assume length $(\eta)=\gamma+1$. If $P(\eta\lceil\gamma)$ is not defined, then $P(\eta)$ is not defined; if $P(\eta\lceil\gamma)$ is defined, then $P(\eta)=P(\eta\lceil\gamma)$ if $\eta(\gamma) \geq \mu$, and otherwise $P(\eta)=P(\eta\lceil\gamma)+1 \bmod 2$.

(c) Assume length $(\eta)=\gamma$ is limit. Let $P(\eta)=0$ if $P(\eta\lceil\beta)=0$ for all $\beta<\gamma$ large enough, and $P(\eta)=1$ if $P(\eta\lceil\beta)=1$ for all $\beta<\gamma$ large enough. Otherwise $P(\eta)$ is left undefined.

$Q(\eta)$ is defined exactly as $P(\eta)$ except that (a) is replaced by

$\left(\mathrm{a}^{\prime}\right)$ if length $(\eta)=0$, then $Q(\eta)=1$.

Claim 1. $(\alpha)$ For all $\eta, P(\eta)$ is defined iff $Q(\eta)$ is defined and in that case $P(\eta)=Q(\eta)+1 \bmod 2$.

( $\beta)$ If $\gamma=\operatorname{length}(\eta)=\operatorname{length}(\xi), \beta<\gamma, P(\eta\lceil\beta)=Q(\xi\lceil\beta)$ and $\eta(\delta)=$ $\xi(\delta)$ for all $\beta \leq \delta<\gamma$, then $P(\eta)$ is defined iff $Q(\xi)$ is defined and in that case $P(\eta)=Q(\xi)$.

$(\gamma)$ If $\gamma=\operatorname{length}(\eta)=\operatorname{length}(\xi)$ and $\{\beta<\gamma \mid \eta(\beta) \neq \xi(\beta)\}$ is finite, then $P(\eta)$ is defined iff $P(\xi)$ is defined.

Proof. Easy. "Claim 1

We let $I \subseteq \kappa^{\leq \alpha}$ be the least set such that

(1) for all $i<\kappa$, if $P\left(f_{i}\right)$ is defined, then $f_{i} \in I$, 
(2) $I$ is closed under initial segments,

(3) if $\eta \in I$, length $(\eta)=\operatorname{length}(\xi)$ and $\{\gamma<\operatorname{length}(\eta) \mid \eta(\gamma) \neq \xi(\gamma)\}$ is finite, then $\xi \in I$.

We define $\mathcal{A}$ and $\mathcal{B}$ so that $\mathcal{A} \cap \kappa^{<\alpha}=\mathcal{B} \cap \kappa^{<\alpha}=I \cap \kappa^{<\alpha}$ and if length $(\eta)=\alpha$, then $\eta \in \mathcal{A}$ (resp. $\eta \in \mathcal{B}$ ) iff $\eta \in I$ and $P(\eta)=0$ (resp. $Q(\eta)=0)$.

Claim 2. $(\mathcal{A}, \mathcal{B})$ is a $\left(\kappa^{+}, \alpha+1\right)$-homogeneous pair of trees, and $|\mathcal{A}|=$ $|\mathcal{B}|=\kappa$.

Proof. We prove only (ii) of the definition of a $\left(\kappa^{+}, \alpha+1\right)$-homogeneous pair of trees as (i) is trivial and (iii) is similar to (ii). So assume $\eta \frown(\delta) \in \mathcal{A}$ and length $(\eta)+1<\alpha$. There are several cases, but they are all similar so we may assume $P(\eta)=Q(\eta)=0$ and $\delta<\mu$. Then $P(\eta \frown(\delta))=Q(\eta \frown(\gamma))$ for all $\gamma<\mu$, and so $\mathcal{A} \uparrow(\eta \frown(\delta)) \cong \mathcal{B} \uparrow(\eta \frown(\gamma))$ by $\operatorname{Claim} 1(\beta)$.

The claim concerning the cardinality of the trees is clear from their definition. Claim 2

Claim 3. $\mathcal{A} \nRightarrow \mathcal{B}$.

Proof. For a contradiction, assume $G: \mathcal{A} \rightarrow \mathcal{B}$ is an isomorphism. Define $g: \kappa^{<\alpha} \rightarrow \kappa$ so that for all $\eta \in \kappa^{<\alpha}$,

(I) if $\eta \notin \mathcal{A}$, then $g(\eta)=\mu$,

(II) if $\eta \in \mathcal{A}$, then $g(\eta)$ is the least $\gamma \geq \mu$ such that the following holds: if $G(\eta \frown(\delta))=G(\eta) \frown\left(\delta^{\prime}\right)$, then $\delta<\gamma$ iff $\delta^{\prime}<\gamma$.

Let $i<\kappa$ be such that $g\left(\left(f_{i}(\delta)\right)_{\delta<\gamma}\right)<f_{i}(\gamma)$ for all $\gamma<\alpha$. Then $f_{i}(\gamma) \geq$ $\mu$ for all $\gamma<\alpha$, and so $P\left(f_{i}\right)=0$, i.e. $f_{i} \in \mathcal{A}$. Now we have two cases:

CASE 1: $\alpha$ is limit. Let $\eta$ be such that $\eta\left\lceil\gamma=G\left(f_{i}\lceil\gamma)\right.\right.$ for all $\gamma<\alpha$. Then $\eta(\gamma) \geq \mu$ for all $\gamma<\alpha$, and so $Q(\eta)=1$, i.e. $\eta \notin \mathcal{B}$, a contradiction.

CAsE 2: $\alpha=\beta+1$. Let $\xi=f_{i} \uparrow \beta$ and $\eta=G(\xi)$. As above, $P(\xi)=0$ and $Q(\eta)=1$. So by the definition of $\mathcal{A}$ and $\mathcal{B}$, and (3) in the definition of $I$, the number of successors of $\xi$ is $\kappa$ and the number of successors of $\eta$ is $\mu$, a contradiction.

Claim 4. $\mathcal{A} \equiv_{\alpha}^{\kappa} \mathcal{B}$.

Proof. We describe the winning strategy of $E$ in the Ehrenfeucht-Fraïssé game $\operatorname{EF}_{\alpha}^{\kappa}(\mathcal{A}, \mathcal{B})$ : We write $\mathcal{A}^{i}$ for the set of all $\eta \in \mathcal{A}$ such that $\operatorname{length}(\eta) \leq i$ and similarly for $\mathcal{B}$. At each move $i<\alpha, E$ chooses a level preserving partial isomorphism $F_{i}$ as follows:

(a) $\bigcup_{j<i} F_{j} \subseteq F_{i}, \operatorname{dom}\left(F_{i}\right) \cup \operatorname{rng}\left(F_{i}\right)$ contains all the elements chosen so far by $A$, and $E$ answers according to $F_{i}$,

(b) $A^{i+1} \subseteq \operatorname{dom}\left(F_{i}\right)$ and $F_{i}\left\lceil\mathcal{A}^{i+1}\right.$ is onto $\mathcal{B}^{i+1}$, 
(c) if length $(\eta)>i+1$ and $\eta \in \operatorname{dom}\left(F_{i}\right)$, then we have $P(\eta \uparrow(i+1))=$ $Q\left(F_{i}(\eta \uparrow(i+1))\right)$; furthermore, if $\eta \in \operatorname{dom}\left(F_{i}\right), j<\operatorname{length}(\eta)$ and $P(\eta\lceil j)=$ $Q\left(F_{i}(\eta)\lceil j)\right.$, then $F_{i}(\eta)(k)=\eta(k)$ for all $j \leq k<\operatorname{length}(\eta)$,

(d) if $\eta \in \operatorname{dom}\left(F_{i}\right)$, length $(\eta) \leq i+1$ and $P(\eta) \neq Q\left(F_{i}(\eta)\right)$, then $F_{i}(\eta)=\eta$.

It is easy to see that player $E$ can find these functions. $\mathbf{M l a i m}$

(ii) Let $\eta_{i}, i<\kappa$, be the enumeration of all $\eta \in \mathcal{A} \cup \mathcal{B}$ such that length $(\eta)=\alpha$. Find $g: \kappa^{<\alpha} \rightarrow \kappa$ such that for all $i<\kappa$ there is $\gamma<\alpha$ for which $g\left(\eta_{i}\lceil\gamma)>\eta_{i}(\gamma)\right.$. Let $\xi_{i}, i<\gamma(*)$, be an enumeration of all $\xi \in \mathcal{A}$ such that $\beta_{i}=$ length $(\xi)<\alpha$. Furthermore assume that the enumeration is such that if $\xi_{i}<\xi_{j}$, then $i<j$.

We define level preserving partial isomorphisms $F_{i}, i<\gamma(*)$, essentially as in Claim 4 above, i.e. so that the following are satisfied:

(1) $\bigcup_{j<i} F_{j} \subseteq F_{i}$,

(2) $\mathcal{A}_{i} \subseteq \operatorname{dom}\left(F_{i}\right)$, where $\mathcal{A}_{i}$ is the set of all $\xi \in \mathcal{A}$ such that for some $j<i$, length $(\xi) \leq \beta_{j}+1$ and $\xi \geq \xi_{j}$,

(3) for all $j<i$, there is $\gamma_{j}<\kappa$ such that $\gamma_{j}>g\left(\xi_{j}\right), \mathcal{A} \uparrow\left(\xi_{j} \frown(\gamma)\right)$ $\subseteq \operatorname{dom}\left(F_{i}\right)$ for all $\gamma<\gamma_{j}, F_{i} \uparrow\left(\mathcal{A} \uparrow\left(\xi_{j} \frown(\gamma)\right)\right)$ is an isomorphism onto $\overline{\mathcal{B}}\left\lceil F_{i}\left(\xi_{j} \frown(\gamma)\right)\right.$ and if $F_{i}\left(\xi_{j} \frown(\delta)\right)=F_{i}\left(\xi_{j}\right) \frown\left(\delta^{\prime}\right)$ then $\delta<\gamma_{j}$ iff $\delta^{\prime}<\gamma_{j}$,

(4) $\operatorname{dom}\left(F_{i}\right)=\mathcal{A}_{i} \cup \bigcup\left\{\mathcal{A} \uparrow\left(\xi_{j} \frown(\gamma)\right) \mid \gamma<\gamma_{j}, j<i\right\}$,

(5) if $\eta \in \mathcal{A}_{i}-\bigcup\left\{\mathcal{A} \uparrow\left(\xi_{j} \frown(\gamma)\right) \mid \gamma<\gamma_{j}, j<i\right\}$, then $F_{i}(\eta)=\eta$.

By induction on $i<\gamma(*)$ using the definition of a $\left(\kappa^{+}, \alpha+1\right)$-homogeneous pair of trees, it is easy to see that $F_{i}$ exists (at limits take unions, which works by (5) above and Definition 2.2(i)).

Let $F=\bigcup_{i<\gamma(*)} F_{i}$. We show that $F$ is an isomorphism from $\mathcal{A}$ to $\mathcal{B}$. By symmetry, it is enough to show that if $\eta \in \mathcal{A}$ is of length $\alpha$, then $\eta \in \operatorname{dom}(F)$. By the choice of $g$, we can find $i<\gamma(*)$ so that $\eta\left\lceil\beta_{i}=\xi_{i}\right.$ and $\eta\left(\beta_{i}\right)<g\left(\xi_{i}\right)$. But then $\eta \in \operatorname{dom}\left(F_{i+1}\right)$.

2.4. Lemma. Let $\kappa>\omega$ and $\lambda \leq \kappa$ be cardinals and $\alpha \leq \kappa$ be a limit ordinal. Assume that for all $\beta<\alpha$ there are $\kappa^{+}, \gamma$-trees $\mathcal{A}_{\beta}$ and $\mathcal{B}_{\beta}$ of power $\kappa$ such that $\gamma<\alpha, \mathcal{A}_{\beta} \equiv_{\beta}^{\lambda} \mathcal{B}_{\beta}$ and $\mathcal{A}_{\beta} \neq \mathcal{B}_{\beta}$. Then there are $\kappa^{+}, \alpha$-trees $\mathcal{A}$ and $\mathcal{B}$ of power $\kappa$ such that $\mathcal{A} \equiv_{\beta}^{\lambda} \mathcal{B}$ for all $\beta<\alpha$ and $\mathcal{A} \neq \mathcal{B}$.

Proof. We assume that all sets $\mathcal{A}_{\beta}$ and $\mathcal{B}_{\gamma}, \beta, \gamma<\alpha$, are disjoint and that $r$ and $e$ do not belong to any of these. We define the universe of $\mathcal{A}$ to consist of those $x$ such that one of (i)-(iii) holds:

(i) $x=r$,

(ii) $x=(\gamma, \beta, \delta, e), \gamma, \beta<\alpha$ and $\delta<\omega$,

(iii) $x=(\gamma, \beta, \delta, y), \gamma, \beta<\alpha, \delta<\omega$ and if $\beta<\gamma$, then $y \in \mathcal{A}_{\beta}$ and otherwise $y \in \mathcal{B}_{\beta}$. 
We order $\mathcal{A}$ as follows: $x \leq x^{\prime}$ if one of (a)-(c) holds:

(a) $x=r$,

(b) $x=(\gamma, \beta, \delta, e)$ and $x^{\prime}=\left(\gamma, \beta^{\prime}, \delta, y\right)$ for some $y$ and $\beta^{\prime} \geq \beta$,

(c) $x=(\gamma, \beta, \delta, y)$ and $x^{\prime}=\left(\gamma, \beta, \delta, y^{\prime}\right)$ for some $y^{\prime} \geq y$.

$\mathcal{B}$ is defined as $\mathcal{A}$ except that (ii) and (iii) are replaced by (ii') and (iii'):

(ii') $x=(\gamma, \beta, \delta, e), \gamma \leq \alpha, \beta<\alpha$ and $\delta<\omega$,

(iii') $x=(\gamma, \beta, \delta, y), \gamma \leq \alpha, \beta<\alpha, \delta<\omega$ and if $\beta<\gamma$, then $y \in \mathcal{A}_{\beta}$ and otherwise $y \in \mathcal{B}_{\beta}$.

Clearly these trees are of power $\kappa$. Let $\mathcal{A}^{*}$ be the subtree of $\mathcal{A}$ which consists of those $x \in \mathcal{A}$ which satisfy (i) or (ii) above. Similarly $\mathcal{B}^{*}$ consists of those $x \in \mathcal{B}$ which satisfy (i) or (ii').

Claim 1. $\mathcal{A} \neq \mathcal{B}$.

Proof. For a contradiction assume that $f$ is an isomorphism from $\mathcal{A}$ to $\mathcal{B}$. Then $f\left\lceil\mathcal{A}^{*}\right.$ is an isomorphism onto $\mathcal{B}^{*}$. This is because for all $x \in \mathcal{A}^{*}$, the height of $\mathcal{A}^{*}\left\lceil x\right.$ is $\alpha$ but for all $y \in \mathcal{B}-\mathcal{B}^{*}$, the height of $\mathcal{B}\lceil y$ is $<\alpha$, and vice versa. So it is easy to find $x=(\gamma, \beta, \delta, y) \in \mathcal{A}$ such that $\beta \geq \gamma, y$ is the root of $\mathcal{B}_{\beta}$ and $f(x)=\left(\alpha, \beta, \delta^{\prime}, y^{\prime}\right)$ for some $\delta^{\prime}$ and $y^{\prime}$ (and so $y^{\prime}$ is the root of $\mathcal{A}_{\beta}$ ). But then $\mathcal{A}_{\beta} \cong \mathcal{B}_{\beta}$, a contradiction.

Claim 2. For all $\beta<\alpha, \mathcal{A} \equiv_{\beta}^{\lambda} \mathcal{B}$.

Proof. Let $\beta<\alpha$ be given. First $E$ chooses an isomorphism $f: \mathcal{A}^{*} \rightarrow \mathcal{B}^{*}$ such that if $x=\left(\gamma, \beta^{\prime}, \delta, e\right), f(x)=\left(\gamma^{\prime}, \beta^{\prime}, \delta^{\prime}, e\right)$ and $\beta^{\prime} \leq \beta$, then $\gamma>\beta^{\prime}$ iff $\gamma^{\prime}>\beta^{\prime}$. If $x=\left(\gamma, \beta^{\prime}, \delta, e\right) \in \mathcal{A}^{*}$, then by $\mathcal{A}_{x}$ we mean the subtree of $\mathcal{A}$ which consists of those $y \in \mathcal{A}-\mathcal{A}^{*}$ such that $x$ is the largest element in $\mathcal{A}^{*}$, which is smaller than $y ; \mathcal{B}_{x}, x \in \mathcal{B}^{*}$, is defined similarly.

Now if $x=\left(\gamma, \beta^{\prime}, \delta, e\right) \in \mathcal{A}^{*}$ and $\beta^{\prime} \leq \beta$, then $\mathcal{A}_{x}$ is isomorphic to $\mathcal{B}_{f(x)}$ and if $\beta^{\prime}>\beta$, then $\mathcal{A}_{x} \equiv_{\beta}^{\lambda} \mathcal{B}_{f(x)}$. So clearly $E$ can play $\beta$ moves in the game $\operatorname{EF}_{\beta}^{\lambda}(\mathcal{A}, \mathcal{B})$. Claim $2 \&$ Lemma 2.4

2.5. Theorem. (i) There are $\omega_{2}, \omega$-trees $\mathcal{A}$ and $\mathcal{B}$ of power $\omega_{1}$ such that $\mathcal{A} ¥ \mathcal{B}$ and $\mathcal{A} \equiv_{n}^{\omega_{1}} \mathcal{B}$ for all $n<\omega$. (In fact, $\mathcal{A} \equiv_{\alpha}^{2} \mathcal{B}$ for all $\alpha<\omega \cdot \omega$.)

(ii) There are $\omega_{3}, \omega \cdot \omega$-trees $\mathcal{A}$ and $\mathcal{B}$ of power $\omega_{2}$ such that $\mathcal{A} \neq \mathcal{B}$ and $\mathcal{A} \equiv_{\alpha}^{\omega_{2}} \mathcal{B}$ for all $\alpha<\omega \cdot \omega$. (In fact, $\mathcal{A} \equiv_{\alpha}^{2} \mathcal{B}$ for all $\alpha<\omega_{1} \cdot \omega \cdot \omega$.)

(iii) Assume $\kappa>\omega_{1}$ is a regular cardinal and $\alpha<\kappa$. Then there are $\kappa^{+2}, \alpha+1$-trees $\mathcal{A}$ and $\mathcal{B}$ of power $\kappa^{+}$such that $\mathcal{A} \neq \mathcal{B}$ and $\mathcal{A} \equiv_{\alpha}^{\kappa^{+}} \mathcal{B}$. (In fact, $\mathcal{A} \equiv_{\kappa \cdot \alpha}^{2} \mathcal{B}$.)

(iv) Assume $\kappa>\omega_{1}$ is a regular cardinal and $\alpha \leq \kappa$ is a limit ordinal. There are $\kappa^{+2}, \alpha$-trees $\mathcal{A}$ and $\mathcal{B}$ of power $\kappa^{+}$such that $\mathcal{A} \neq \mathcal{B}$ and $\mathcal{A} \equiv_{\beta}^{\kappa^{+}} \mathcal{B}$ for all $\beta<\alpha$. (In fact, $\mathcal{A} \equiv_{\beta}^{2} \mathcal{B}$ for all $\beta<\kappa \cdot \alpha$. $)$ 
(v) If $\square_{\omega_{1}}$ holds, then there are $\omega_{3}, \omega_{1}$-trees $\mathcal{A}$ and $\mathcal{B}$ of power $\omega_{2}$ such that $\mathcal{A} \neq \mathcal{B}$ and $\mathcal{A} \equiv_{\alpha}^{\omega_{2}} \mathcal{B}$ for all $\alpha<\omega_{1}$. (In fact, $\mathcal{A} \equiv_{\alpha}^{2} \mathcal{B}$ for all $\alpha<\omega_{1} \cdot \omega_{1}$.)

(vi) If $*_{\alpha}^{\omega_{1}}$ holds for all $\alpha<\omega_{1}$, then there are $\omega_{2}, \omega_{1}$-trees $\mathcal{A}$ and $\mathcal{B}$ of power $\omega_{1}$ such that $\mathcal{A} ¥ \mathcal{B}$ and $\mathcal{A} \equiv_{\alpha}^{\omega_{1}} \mathcal{B}$ for all $\alpha<\omega_{1}$.

Proof. Immediate by Lemmas 2.3 and 2.4 and Conclusion 1.8 .

Notice that by Theorem 2.1, in Theorem 2.5(i), " $\mathcal{A} \equiv_{\alpha}^{2} \mathcal{B}$ for all $\alpha<\omega \cdot \omega$ " and in (v) " $\mathcal{A} \equiv_{\alpha}^{2} \mathcal{B}$ for all $\alpha<\omega_{1} \cdot \omega_{1}$ " are the best possible and in (iv), if $\kappa=\lambda^{+}, \lambda$ regular, then " $\mathcal{A} \equiv_{\alpha}^{2} \mathcal{B}$ for all $\beta<\kappa \cdot \alpha$ " is often the best possible (for $\kappa$ many $\alpha \leq \kappa, \lambda \cdot \alpha=\alpha$ ).

2.6. REMARK. By [NS] and [Sh5] the following holds (for linear orderings this is implicit in [NS]): Assume $\kappa=\mu^{+}$and $\mu$ is regular. Then for all countable unstable $T$, there are $\mathcal{A}, \mathcal{B} \models T$ of power $\kappa$ such that $\mathcal{A} ¥ \mathcal{B}$ and $\mathcal{A} \equiv_{\mu}^{\kappa} \mathcal{B}$. This can be seen as follows: Choose a linear ordering $\theta$ so that $|\theta| \leq \kappa, \theta \cong \theta \cdot(\alpha+1)$ for $\alpha \leq \mu, \theta \cong \theta \cdot \mu+\theta \cdot \omega_{1}^{*}$ and $\operatorname{cf}\left(\theta^{*}\right)=\omega$, where by $\omega_{1}^{*}$ and $\theta^{*}$ we denote the inverse of $\omega_{1}$ and $\theta$, respectively. Then by appplying the $\Phi$-model construction from [NS], we get linear orders $\Phi(A)$, $A \subseteq\{\alpha<\kappa \mid \operatorname{cf}(\alpha)=\mu\}$, such that for all $A$ and $B, \Phi(A) \equiv_{\mu}^{\kappa} \Phi(B)$ and $\operatorname{inv}_{\omega}^{1}(\Phi(A))=\operatorname{inv}_{\omega}^{1}(\Phi(B))$ iff the symmetric difference of $A$ and $B$ is not stationary. Now the claim follows by using [Sh5], Chapter 3.

Notice that in the remark above, if $I\left[\kappa^{+}\right]$is improper, then $\Phi(A) \not \equiv_{\mu+\omega+1}^{2}$ $\Phi(B)$ (unless $\Phi(A) \cong \Phi(B)$; see $[\mathrm{HHR}])$.

\section{References}

[Ba] J. E. Baumgartner, Iterated forcing, in: A. Mathias (ed.), Surveys in Set Theory, London Math. Soc. Lecture Note Ser. 87, Cambridge Univ. Press, 1978, 1-59.

[EFS] P. Eklof, M. Foreman and S. Shelah, On invariants for $\aleph_{1}$-separable groups, Trans. Amer. Math. Soc. 347 (1995), 4385-4402.

[Hu] T. Huuskonen, Observations about Scott and Karp trees, Ann. Pure Appl. Logic 76 (1995), 201-230.

[HHR] T. Huuskonen, T. Hyttinen and M. Rautila, On the $\kappa$-cub game on $\lambda$ and $I[\lambda]$, Arch. Math. Logic 38 (1999), 549-557.

[HS] T. Hyttinen and S. Shelah, Constructing strongly equivalent nonisomorphic models for unsuperstable theories, part A, J. Symbolic Logic 59 (1994), 984-996.

[HST] T. Hyttinen, S. Shelah and H. Tuuri, Remarks on strong nonstructure theorems, Notre Dame J. Formal Logic 34 (1993), 157-168.

[HT] T. Hyttinen and H. Tuuri, Constructing strongly equivalent nonisomorphic models for unstable theories, Ann. Pure Appl. Logic 52 (1991), 203-248.

[KS] M. Kojman and S. Shelah, The universality spectrum of stable unsuperstable theories, ibid. 58 (1992), 57-72.

[MO] A. Mekler and J. Oikkonen, Abelian groups with no invariants, J. Pure Appl. Algebra 87 (1993), 51-59. 
[NS] M. Nadel and J. Stavi, $L_{\infty \lambda}$-equivalence, isomorphism and potential isomorphism, Trans. Amer. Math. Soc. 236 (1978), 51-74.

[Sh1] S. Shelah, Existence of many $L_{\infty, \lambda}$-equivalent, nonisomorphic models of $T$ of power $\lambda$, Ann. Pure Appl. Logic 34 (1987), 291-310.

[Sh2] - Reflecting stationary sets and successors of singular cardinals, Arch. Math. Logic 31 (1991), 25-54.

[Sh3] -, Cardinal Arithmetics, Oxford Logic Guides 29, Clarendon Press, Oxford, 1994.

[Sh4] - , Proper and Improper Forcing, Perspectives in Mathematical Logic, Springer, Heidelberg, 1998.

[Sh5] - Non-structure Theory, to appear.

[Tu] H. Tuuri, Infinitary languages and Ehrenfeucht-Fraïssé games, doctoral thesis, University of Helsinki, 1990.

Department of Mathematics

P.O. Box 4

00014 University of Helsinki

Finland

E-mail: thyttine@cc.helsinki.fi

Received 28 February 2000;

in revised form 13 November 2000 